\title{
Implications of bleeding in acute coronary syndrome and percutaneous coronary intervention
}

This article was published in the following Dove Press journal:

Vascular Health and Risk Management

25 August 2011

Number of times this article has been viewed

\author{
Phuong-Anh Pham' \\ Phuong-Thu Pham ${ }^{2}$ \\ Phuong-Chi Pham ${ }^{3}$ \\ Jeffrey $M$ Miller \\ Phuong-Mai Pham 5 \\ Son $\vee$ Pham $^{6}$
}

'Department of Medicine, Division of Cardiovascular Diseases,

VA Medical Center and University of Tennessee Health Science Center, Memphis, TN, USA; ${ }^{2}$ Department of Medicine, Nephrology Division, Kidney Transplant Program, David Geffen School of Medicine at UCLA; ${ }^{3}$ Department of Medicine, Nephrology Division, ${ }^{4}$ Department of Medicine, Hematology-Oncology Division, UCLA-Olive View Medical Center and David Geffen School of Medicine at UCLA; ${ }^{5}$ Department of Medicine, Greater Los Angeles VA Medical Center and David Geffen School of Medicine at UCLA, Los Angeles, CA, USA; ${ }^{6}$ Department of Medicine, Division of Cardiovascular Diseases, Bay Pines VA Medical Center, Bay Pines, FL, USA
Correspondence: Phuong-Thu Pham Department of Medicine, Nephrology Division, Kidney Transplant Program, David Geffen School of Medicine at UCLA, Los Angeles, CA 90095 , USA

Tel +I 3107941757

Email ppham@mednet.ucla.edu

\begin{abstract}
The advent of potent antiplatelet and antithrombotic agents over the past decade has resulted in significant improvement in reducing ischemic events in acute coronary syndrome (ACS). However, the use of antiplatelet and antithrombotic combination therapy, often in the settings of percutaneous coronary intervention (PCI), has led to an increase in the risk of bleeding. In patients with non-ST elevation myocardial infarction treated with antithrombotic agents, bleeding has been reported to occur in $0.4 \%-10 \%$ of patients, whereas in patients undergoing PCI, periprocedural bleeding occurs in $2.2 \%-14 \%$ of cases. Until recently, bleeding was considered an intrinsic risk of antithrombotic therapy, and efforts to reduce bleeding have received little attention. There have been increasing data demonstrating that bleeding is associated with adverse outcomes, including myocardial infarction, stroke, and death. Therefore, it is imperative to optimize patient outcomes by adopting pharmacological and nonpharmacological strategies to minimize bleeding while maximizing treatment efficacy. In this paper, we present a review of the bleeding classifications used in large-scale clinical trials in patients with ACS and those undergoing PCI treated with antiplatelets and antithrombotic agents, adverse outcomes, particularly mortality associated with bleeding complications, and suggested predictive risk factors. Potential mechanisms of the association between bleeding and mortality and strategies to reduce bleeding complications are also discussed.
\end{abstract}

Keywords: bleeding risk, antiplatelets, antithrombotics, acute coronary syndrome, percutaneous coronary intervention

\section{Bleeding classifications: historical perspectives}

The TIMI and GUSTO scales were the first bleeding classifications developed to measure short-term bleeding associated with thrombolytic therapy in patients with ST elevation myocardial infarction (STEMI). The TIMI classification was a laboratorybased scale that categorized bleeding as major or minor. ${ }^{1}$ Major bleeding was defined as any intracranial bleeding or a reduction in hemoglobin of $\geq 5 \mathrm{~g} / \mathrm{dL}$ (or a $>15 \%$ decrease in hematocrit). Minor bleeding was defined by an observed blood loss and a drop in hemoglobin of 3-5 g/dL (or in hematocrit of 10\%-15\%), spontaneous gross hematuria or hematemesis $(>120 \mathrm{~mL})$, even if the hemoglobin or hematocrit drop was $<3$ g or $<10 \%$, respectively, or an unobserved loss of $\geq 4 \mathrm{~g} / \mathrm{dL}$ in hemoglobin or $\geq 12 \%$ in hematocrit.

The GUSTO classification was based on clinical events that classified bleeding as severe/life-threatening, moderate, or mild. ${ }^{2}$ Severe bleeding was defined as intracranial bleeding or bleeding which resulted in substantial hemodynamic compromise requiring intervention. Moderate bleeding was defined by the need for blood transfusion 
but without hemodynamic insult, and minor bleeding was defined by bleeding which did not require transfusion or cause hemodynamic instability.

With the advent of more potent antithrombotic agents and American College of Cardiology/American Heart Association guidelines for long-term oral antiplatelet therapy in patients with acute coronary syndrome (ACS) and those undergoing percutaneous coronary intervention (PCI), the TIMI and GUSTO scales may have limited applicability to current clinical trials. It should be noted that the TIMI definition was developed to quantify bleeding complications in patients receiving thrombolytic therapy for STEMI and may underestimate the impact of bleeding complications on mortality in patients undergoing planned or urgent PCI. Due to the limitations of these scales, over the last half decade clinical investigators have devised study-specific bleeding definitions to assess patients' risks and outcomes, such as those defined by the ACUITY and REPLACE-2 trials (discussed below). ${ }^{3,4}$

Noncoronary artery bypass graft (CABG) ACUITY-scale major bleeding was defined as intracranial or intraocular bleeding, access site hemorrhage requiring intervention, hematoma $\geq 5 \mathrm{~cm}$ in diameter, reduction in hemoglobin of $\geq 3 \mathrm{~g} / \mathrm{dL}$ with an overt source of bleeding or $\geq 4 \mathrm{~g} / \mathrm{dL}$ and no source identified, reoperation for bleeding, or blood product transfusion. Analysis of the ACUITY trial demonstrated that major bleeding is a powerful predictor of 30-day mortality in patients with ACS managed invasively (odds ratio [OR] $7.55, P<0.0001$ ). Independent predictors of major bleeding were advanced age, female gender, diabetes, hypertension, renal insufficiency, anemia, no prior PCI, cardiac biomarker elevation, $\mathrm{ST}$ segment elevation $\geq 1 \mathrm{~mm}$, and treatment with heparin plus glycoprotein IIb/IIIa inhibitor (GPI) versus bivalirudin monotherapy.

The REPLACE-2 trial classified bleeding as major and minor based on both adverse clinical events and laboratory values. Major hemorrhage was defined as intracranial, intraocular, or retroperitoneal, overt blood loss with a $>3 \mathrm{~g} / \mathrm{dL}$ decrease in hemoglobin, any hemoglobin decrease $>4 \mathrm{~g} / \mathrm{dL}$, or transfusion of $\geq 2$ units of blood products. Minor hemorrhage was defined as overt bleeding that did not meet the criteria for major bleeding. Major hemorrhage as defined by the REPLACE-2 study protocol was found to be an independent predictor of mortality in patients undergoing elective or urgent PCI. Independent baseline predictors of major hemorrhage included advanced age, female gender, impaired creatinine clearance, and anemia (defined using World Health Organization criteria of hemoglobin $<13 \mathrm{~g} / \mathrm{dL}$ in men and $<12 \mathrm{~g} / \mathrm{dL}$ in women) whereas independent periprocedural predictive factors included treatment with heparin plus GPI, increased procedural duration, provisional use of GPI, increased time to sheath removal, length of intensive care unit stay, and use of an intra-aortic balloon pump (all $P<0.05) .^{5}$

Until recently, there has been no consensus on the endpoint definitions of bleeding in patients with ACS or those undergoing PCI. In February 2010, the Bleeding Academic Research Consortium consisting of leaders in the field of cardiovascular disease and experts from the academic research organizations, the Food and Drug Administration, the National Institutes of Health, and the pharmaceutical and cardiovascular device manufacturers set forth a standardized Bleeding Academic Research Consortium definition of bleeding. The proposed bleeding definition consisted of five types, from 0 to 5 . Type 0 was defined as no evidence of bleeding. Type 1 was defined as bleeding which is not actionable and does not cause the patient to seek unscheduled performance of studies, hospitalization, or treatment by a health care professional. Type 2 was defined as any clinically overt sign of hemorrhage that is actionable but does not meet criteria for type 3, type 4 (CABG-related), or type 5 (fatal bleeding). The bleeding must require diagnostic studies, hospitalization, or treatment by a health care professional. Type 3 was defined as clinical, laboratory, and/or imaging evidence of bleeding with specific health care provider responses. Type 4 was defined as CABG-related bleeding and type 5 was defined as fatal bleeding. It is noteworthy that the proposed bleeding definitions were based on expert consensus rather than clinical data. Validation of the consensus definitions is still needed from future cardiovascular clinical trials. ${ }^{6,7}$

Currently, the American College of Cardiology task force on clinical data standards recommends using the TIMI classification as the standard for classifying bleeding events. Nonetheless, in a pooled analysis of the PURSUIT $(\mathrm{n}=10,798)$ and PARAGON B $(\mathrm{n}=5225)$ trials to determine the association between the severity of TIMI and GUSTO bleeding and 30-day and six-month death or myocardial infarction, Rao et al demonstrated that while both GUSTO and TIMI bleeding scales identified patients at risk for adverse outcomes, when both bleeding definitions were included in the same model, the stepwise increase in the risk with worsening GUSTO bleeding persisted, whereas the association between any level of TIMI bleeding and outcome was no longer significant. ${ }^{8}$ The results of the study suggested that clinically evident bleeding added prognostic value to laboratory parameters. 
In summary, bleeding classification appears to have a better prognostic value when both clinical and laboratory data are incorporated. It has generally been accepted that any significant bleeding episode involves intracranial or intraocular bleeding, hemodynamic compromise requiring blood transfusions, observed blood loss with a hemoglobin drop of at least $3 \mathrm{~g} / \mathrm{dL}$, or access site complications requiring surgical intervention.

The Bleeding Academic Research Consortium definitions of bleeding are summarized in Table 1. The definitions of major bleeding in large-scale clinical trials (discussed in this article) are summarized in Table 2.

\section{Adverse outcomes associated with bleeding in ACS and $\mathrm{PCI}$}

Bleeding is an important safety concern for patients with ACS and those undergoing PCI who are managed with multiple antithrombotic agents. The effect of bleeding was thought to be acute, and when treated accordingly, had no long-term impact. However, over the last half decade, there has been increasing evidence demonstrating an association between bleeding and short-term and long-term adverse cardiovascular events, most importantly, mortality. An overview of the literature on the impact of bleeding on short-term and long-term outcomes, particularly mortality in patients with
ACS and those undergoing PCI, is presented followed by a brief discussion of the authors'perspectives.

Rao et al were among the first to report the association between bleeding and mortality. In a meta-analysis of four multicenter, randomized clinical trials to determine the association between bleeding severity as measured by the GUSTO scale and mortality rates in patients with NSTEMI-ACS, Rao et al demonstrated stepwise increases in 30-day and six-month mortality as bleeding severity increased. ${ }^{9}$ Clinical data were obtained from the GUSTO IIb $(\mathrm{n}=8011)$, PURSUIT $(\mathrm{n}=10,948)$, and PARAGON A and $B(n=2282$ and $n=5225$, respectively) trials. Of more than 26,000 patients studied, $27.6 \%$ had at least one bleeding event during the index hospitalization. The proportions of patients with mild, moderate, and severe bleeding were $16.6 \%, 9.8 \%$, and $1.2 \%$, respectively. Older age, female gender, the presence of cardiac risk factors, inhospital treatment with hirudin, eptifibatide, or lamifiban, and the need for invasive cardiac procedures were seen more frequently in patients who had a bleeding event compared with those who did not $(P<0.001)$. There were stepwise increases in the adjusted hazards of 30-day and six-month mortality as bleeding severity increased (hazards ratio [HR] for 30-day mortality categorized by bleeding severity were 1.6 [1.3-1.9], 2.7 [2.3-3.4], and 10.6 [8.3-13.6] for mild, moderate, and

Table I Bleeding Academic Research Consortium bleeding definitions

Type 0 No bleeding

Type I Bleeding that is not actionable and does not cause the patient to seek unscheduled performance of studies, hospitalization, or treatment by a health care professional; may include episodes leading to self-discontinuation of medical therapy by the patient without consulting a health care professional

Type 2 Any overt, actionable sign of bleeding (eg, more bleeding than would be expected for a clinical circumstance, including bleeding found by imaging alone) that does not fit the criteria for type 3, 4, or 5 but does meet at least one of the following criteria: requiring nonsurgical, medical intervention by a health care professional; leading to hospitalization or increased level of care; or prompting evaluation

Type 3

Type 3a Overt bleeding plus hemoglobin drop of 3-5 g/dL* (provided hemoglobin drop is related to bleed) Any transfusion with overt bleeding

Type 3b Overt bleeding plus hemoglobin drop $\geq 5 \mathrm{~g} / \mathrm{dL}^{*}$ (provided hemoglobin drop is related to bleed) Cardiac tamponade

Bleeding requiring surgical intervention for control (excluding dental/nasal/skin/hemorrhoid)

Bleeding requiring intravenous vasoactive agents

Type 3c Intracranial bleeding (does not include microbleeds or hemorrhagic transformation, does include intraspinal) Subcategories confirmed by autopsy or imaging or lumbar puncture Intraocular bleed compromising vision

Type 4 Coronary artery bypass graft-related bleeding

Perioperative intracranial bleeding within 48 hours

Reoperation after closure of sternotomy for the purpose of controlling bleeding

Transfusion of $\geq 5 \cup$ whole blood or packed red blood cells within a 48 -hour period ${ }^{\dagger}$

Chest tube output $\geq 2 \mathrm{~L}$ within a 24 -hour period

Type 5 Fatal bleeding

Type 5a Probable fatal bleeding; no autopsy or imaging confirmation but clinically suspicious

Type 5b Definite fatal bleeding; overt bleeding or autopsy or imaging confirmation

Notes: *Corrected for transfusion ( $\mathrm{U}$ packed red blood cells or I $\mathrm{U}$ whole blood = I g/dL hemoglobin); ${ }^{\dagger}$ cell saver products are not counted. 


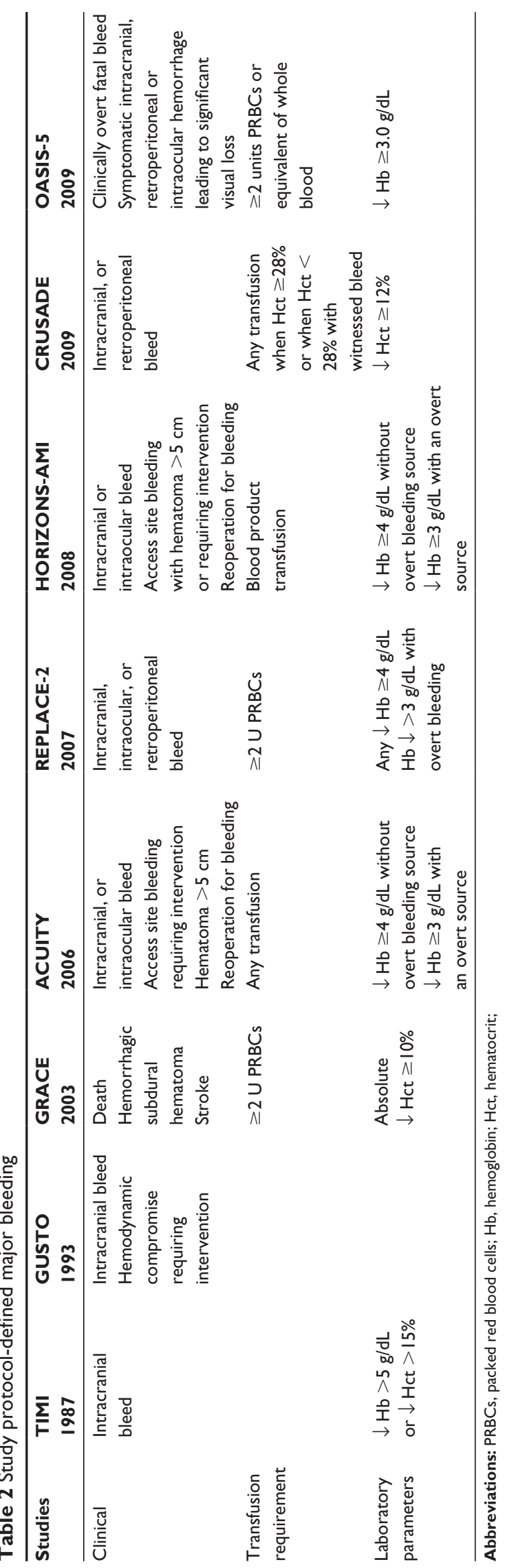

severe bleeding, respectively). The corresponding HR for six-month mortality were 1.4 (1.2-1.6), 2.1 (1.8-2.4), and 7.5 (6.1-9.3), respectively. The association between severity of bleeding and adverse outcomes remained after adjustment for potential confounding factors, including blood transfusions and after censoring for $\mathrm{CABG}$ and for procedure-related and nonprocedure-related bleeds. The results of the study suggested that the GUSTO bleeding classification can identify a subset of patients who are at risk for short-term and longterm adverse events.

Subsequent studies by Eikelboom et al similarly demonstrated a strong temporal and dose-related association between bleeding and death in ACS patients without persistent ST segment elevation. ${ }^{10}$ Data were obtained from the OASIS-1, OASIS-2, and CURE trials (OASIS- $1 \mathrm{n}$ about 11,500; OASIS- $2 \mathrm{n}=10,141$; and CURE $\mathrm{n}=12,562$ ). Major bleeding was defined in each study as significantly disabling, transfusion requirement of $\geq 2$ units of packed cells, or life-threatening. Major bleeding was independently associated with an increased risk of death during the first 30 days (HR 5.37, $P<0.0001$ ). In contrast, the HR was weaker between 30 days and six months (HR 1.54, $P=0.047$ ). Independent baseline predictors of major bleeding included older age, diabetes, prior stroke, lower systolic and diastolic blood pressures, higher serum creatinine, and ST segment changes on electrocardiography. The associations between major bleeding were consistent across subgroups according to cointerventions during hospitalization (treatment with a GPI, unfractionated heparin, low-molecular-weight heparin, fibrinolysis, treatment with oral anticoagulants or the need for undergoing coronary angiography, $\mathrm{CABG}$, or intra-aortic balloon pump). Further analysis demonstrated a dose relationship between severity of bleeding and increasing risk of death (minor less than major less than life-threatening, $P_{\text {trend }}=0.009$ ). A similar association was observed between major bleeding and increased risk of ischemic events (myocardial infarction, stroke) at 30 days and between 30 days and six months.

The OASIS-5 trial previously demonstrated that fondaparinux was similar to enoxaparin in reducing the risk of ischemic events in patients with non-ST elevation ACS at nine days while substantially reducing the risk of major bleeding. ${ }^{11}$ More importantly, reduced major bleeding was associated with improved long-term morbidity and mortality. In a study to determine the incidence and outcomes after bleeding in more than 20,000 patients enrolled in the OASIS- 5 trial, Budaj et al demonstrated that fondaparinux reduced bleeding compared with enoxaparin, but more 
importantly, the reduced risk of bleeding was associated with reduced mortality. ${ }^{12}$ Of the 57 excess deaths between the two groups at 30 days, $66.7 \%$ of the deaths were patients in the enoxaparin group who experienced bleeding (fatal, major, and minor). Similarly, of the 64 excess deaths between the two groups at 180 days, $95.3 \%$ of the deaths occurred in patients from the enoxaparin group who experienced bleeding (fatal, major, and minor). Within each treatment group, patients who had more severe bleeding had a higher risk of death (major versus minor). Analysis of the relationship between major bleeding and outcomes at 30 days in the overall patient population revealed a nearly four-fold increase in the composite endpoints of death, myocardial infarction, or stroke $(21.8 \%$ versus $6.2 \%$, HR $3.99,95 \%$ confidence interval [CI] 3.30-4.82). A similar increase in each of the individual components of the composite outcome was also observed, ie, death $(8.4 \%$ versus $2.7 \%$, HR $3.46,95 \%$ CI $2.60-4.60)$, myocardial infarction $(8.3 \%$ versus $3.4 \%$, HR $4.39,95 \%$ CI $3.45-5.59)$, and stroke (3.0\% versus $0.7 \%$, HR $4.66,95 \%$ CI $2.83-7.65, P<0.0001)$. The magnitude of association was attenuated at 180 days but remained significant (approximately three-fold increase for the composite endpoints and for each individual component, $P<0.0001$ ). Minor bleeding was also independently associated with an increased risk of death, myocardial infarction, and stroke as well as the composite endpoints of death/myocardial infarction/stroke, at 30 and 180 days. The results of the study showed an independent, temporal, and dose-related association between bleeding and the composite endpoints of death/myocardial infarction/stroke, as well as its individual components. Patients who bled were older, more likely to be female, more likely to have a history of diabetes, hypertension, stroke, peripheral vascular disease, heart failure, higher serum creatinine, lower baseline hemoglobin, or ST segment changes on the electrocardiogram at presentation. In addition, they were more likely to be treated with antithrombotic and antiplatelet medications, and to undergo procedures (coronary angiography, PCI, or CABG). The investigators concluded that bleeding in patients with ACS is a powerful determinant of fatal and nonfatal outcomes. Reducing the risk of bleeding using a safer anticoagulation strategy during the first nine days is associated with substantial reduction in morbidity and mortality.

Although analyses from large-scale, randomized, controlled trials suggest that bleeding independently predicts mortality in ACS and PCI, the relative predictive value of bleeding compared with other comorbid factors associated with mortality remains to be elucidated.
In an analysis of findings from the GRACE registry study, Spencer et al demonstrated that the risk of hospital mortality associated with bleeding was much lower than that reported in randomized controlled trials. ${ }^{13}$ The authors speculated that although bleeding may be causally related to adverse outcome in the "real-world" setting, it is often merely a marker for patients at a higher risk for adverse outcomes. Of more than 40,000 patients with acute myocardial infarction (53\% STEMI, 47\% NSTEMI) included in the GRACE study, $2.8 \%$ experienced major bleeding during their index hospitalization. Nearly half of these events (49\%) occurred on the day of admission or on the day after admission. Patients with bleeding were more likely to die during hospitalization (HR 1.9, 95\% CI 1.6-2.2) but not after discharge (HR 0.8, 95\% CI 0.6-1.0). Analysis between bleeding and outcomes among patients who bled, regardless of whether fibrinolytic therapy was given, similarly demonstrated an increased risk of inhospital death in patients who developed a major bleed. Of the 5931 patients who received fibrinolytic therapy, $3.1 \%$ had a major bleeding episode. Their adjusted risk of inhospital death compared with those without a bleeding episode was three times higher (HR 3.3, 95\% CI 2.3-4.7). Among patients who bled but did not receive fibrinolytic therapy, the risk of inhospital death was attenuated but remained significant (HR 1.7, 95\% CI 1.4-2.0). Despite the strong association between bleeding and inhospital mortality, it is noteworthy that, among patients who suffered major bleeding within the first hospital day, mortality rates were higher among those who discontinued aspirin, thienopyridines, or unfractionated heparin compared with those who bled but continued to be treated with these agents after the first day, ie, aspirin (OR 7.55, 95\% CI 4.43-12.88), thienopyridines (OR 8.91, 95\% CI 4.39-18.2), and heparin (OR 1.91, 95\% CI 1.09-3.36). Hence it is speculated that discontinuation of antiplatelet and antithrombotic therapies might explain in part the increased mortality rates observed in patients who experienced bleeding complications.

In a study to determine the predictors of major bleeding and the impact of major bleeding on 30-day outcomes including mortality in patients with ACS, Manoukian et al demonstrated that major bleeding is a powerful independent predictor of 30-day mortality in patients with ACS managed invasively. ${ }^{3}$ Data were obtained from the randomized, openlabeled, Phase III ACUITY trial. ${ }^{14}$ Study subjects consisted of nearly 14,000 patients with ACS in whom urgent or early intervention was planned. Of the 13,819 patients studied, $4.7 \%$ experienced major bleeding. The use of bivalirudin 
alone compared with unfractionated heparin plus GPI was associated with a noninferior rate of composite ischemic endpoint (death, myocardial infarction, unplanned revascularization for ischemia) but significantly reduced the rates of major bleeding and, hence, net clinical outcome. Patients with major bleeding had higher 30-day mortality rates, composite ischemia, and stent thrombosis compared with those without major bleeding ( $P<0.0001$ for each analysis). More importantly, major bleeding was found to be an independent predictor of 30-day mortality (OR 7.55, 95\% CI 4.68-12.18, $P<0.0001)$. Older age, lower body weight, female gender, diabetes, hypertension, anemia, renal insufficiency, elevated cardiac biomarkers, and ST segment deviation of $\geq 1 \mathrm{~mm}$ were more frequently observed in patients who experienced major bleed. They were also more frequently given a GPI, and more likely to undergo PCI rather than CABG, or be assigned to medical therapy. Knowledge of these findings might be useful to reduce bleeding risk and improve outcomes in ACS.

It has been suggested that the relative value of bleeding in predicting mortality can be assessed by determining the overall contribution of bleeding to mortality. Early results of the ACUITY trial demonstrated that both major bleeding and myocardial infarction were independent predictors of 30-day mortality. Mehran et al subsequently evaluated the risk of each of these events on the magnitude and timing of mortality at one-year follow-up. ${ }^{15}$ Of more than 13,000 patients with moderate and high-risk ACS enrolled in the ACUITY trial, 5.1\% had a myocardial infarction and 4.7\% had a major bleed within 30 days of randomization. Patients who had a major bleed had a 3.5-fold increased risk of one-year mortality compared with those who did not bleed, whereas patients who had a myocardial infarction had a 3.1-fold increased risk compared with those who did not have a myocardial infarction. Although the risk was slightly greater for patients who had a major bleed compared with those who had a myocardial infarction, it is noteworthy that the risk of death in patients who experienced a myocardial infarction was time-dependent, with the risk declining rapidly after 30 days (HR 17.7, 95\% CI 10.8-28.7, day 0-1; HR 1.4, 95\% CI 0.9-2.1, days 31+). In contrast, the risk of death in patients who experienced a major bleed did not vary in the first 30 days, and although the risk slightly decreased after day 30, it remained fairly steady over time throughout one year. The results of the study demonstrated a strong association of both major bleeds and myocardial infarction with mortality in the first year after ACS. However, myocardial infarction was correlated with a dramatic increase in short-term risk, whereas major bleed correlated with a more prolonged mortality risk.

The REPLACE-2 trial was the first large-scale randomized, double-blind, active controlled trial comparing inhospital major bleeding rates associated with bivalirudin and provisional GPI during PCI versus standard practice of low-dose heparin plus planned GPI. ${ }^{4}$ Of 6010 patients enrolled in the study, 3.2\% experienced major hemorrhage as defined by the REPLACE-2 study protocol. Independent baseline predictors of major hemorrhage included advanced age, female gender, impaired creatinine clearance, and anemia (defined as hemoglobin $<13 \mathrm{~g} / \mathrm{dL}$ in men and $<12 \mathrm{~g} / \mathrm{dL}$ in women), whereas independent periprocedural predictive factors included treatment with heparin plus GPI, increased procedural duration, provisional use of GPI, increased time to sheath removal, length of intensive care unit stay, and use of an intra-aortic balloon pump. Patients with major bleeding compared with those without major bleeding had significantly higher rates of mortality at 30 days $(5.1 \%$ versus $0.2 \%, P<0.001)$, six months $(6.7 \%$ versus $1.0 \%$, $P<0.001)$, and one year (8.7\% versus $1.9 \%, P<0.0001)$. Multivariate analysis demonstrated that periprocedural major bleeding was an independent predictor of one-year mortality (OR 2.66, 95\% CI 1.44-4.92, $P=0.002)^{5}$

In a retrospective analysis to investigate the incidence, predictors, and impact of periprocedural bleeding and blood transfusion in $>10,000$ patients undergoing PCI, Kinnaird et al demonstrated that major bleeding was an independent predictor of inhospital death after PCI (OR 3.5, $P=0.0001)$ but not after one year, whereas transfusion was an independent predictor of inhospital and one-year mortality (OR 2.0, $P<0.002$; OR 1.9, $P<0.0001) .{ }^{16}$ Major and minor bleeding were defined by the TIMI criteria, and no bleeding was defined as a hematocrit decrease of $<10$ points with no clinical bleeding. Of the study sample, major bleeding occurred in $5.4 \%$, minor bleeding in $12.7 \%$, and no bleeding in $81.9 \%$. Patients who experienced bleeding had higher inhospital mortality (7.5\% major, $1.8 \%$ minor, and $0.6 \%$ no bleeding) and higher one-year mortality (17.2\% versus $9.1 \%$ versus $5.5 \%$ ) in a stepwise fashion with bleeding severity. On multivariate analysis, the increased mortality rates remained after one year for each bleeding category $(P<0.001)$. Patients who bled and received transfusions compared with those who did not had significantly higher inhospital mortality, regardless of their bleeding category (10.6\% versus $5.1 \%$ for major bleeding, $6.8 \%$ versus $0.9 \%$ for minor bleeding, $10.3 \%$ versus $0.4 \%$ for no bleeding; $P<0.001$ for all comparisons). Furthermore, there was a significant association between the 
number of units transfused and one-year mortality (OR 1.47 per unit transfused, $P<0.001)$. The use of an intra-aortic balloon pump (OR 3.0, $P<0.0001$ ), procedural hypotension (OR 2.9, $P<0.001$ ), and age $>80$ years (OR 1.9 compared with age $<50$ years, $P=0.001)$ were the strongest predictors for major bleeding.

In a meta-analysis of four randomized clinical trials (ISAR-REACT, -SWEET, -SMART-2, REACT-2) to investigate the relationship between bleeding (defined according to the TIMI criteria) within 30 days post-PCI and one-year mortality and to assess the appropriateness of inclusion of periprocedural bleeding in a quadruple composite endpoint, Ndrepepa et al demonstrated that bleeding in the first 30 days after PCI was as strongly and independently associated with mortality as the 30-day occurrence of other commonly used components of the composite endpoints in PCI studies, such as myocardial infarction, urgent revascularization, or death. ${ }^{17}$ Study subjects consisted of $>5000$ patients with a wide range of coronary artery disease (from lower-risk patients undergoing elective PCI to higher-risk patients with an NSTEMI) who were randomly assigned to receive abciximab after pretreatment with $600 \mathrm{mg}$ clopidogrel or placebo as a control. The 30-day occurrence of bleeding (HR 2.96, $P<0.001$ ), myocardial infarction (HR 2.29, $P<0.001$ ), and urgent revascularization (HR $2.49, P=0.019$ ) independently predicted one-year mortality. It is noteworthy that not only major but minor bleeding was also associated with oneyear mortality (OR 5.00 versus patients without bleeding, $P<0.001)$. The results of the study support the appropriateness of including periprocedural bleeding in a quadruple composite of primary endpoints to assess outcomes in patients undergoing PCI. Overall, patients who died had a worse cardiovascular risk profile, including older age ( 74.3 versus 66.3 years), lower body mass index, history of diabetes, elevated cardiac enzymes and serum creatinine, multivessel disease, and reduced left ventricular function (ejection fraction $51.0 \%$ versus $59.0 \%, P<0.001$ for each variable).

Results from the HORIZONS-AMI consisting of 3602 STEMI patients undergoing primary PCI who were randomized to receive bivalirudin monotherapy and provisional GPI or heparin plus GPI demonstrated that bivalirudin monotherapy resulted in significantly reduced 30-day rates of major bleeding and net adverse clinical events $(9.2 \%$ versus $12.1 \%$; relative risk [RR] $0.76, P=0.005$ ), compared with heparin plus GPI (abciximab or eptifibatide) owing to a lower rate of major bleeding ( $4.9 \%$ versus $8.3 \%$, respectively, $P<0.001) .{ }^{18}$ Similarly, 30-day rates of death from cardiac causes (RR 0.62, $P=0.03)$ and death from all causes (RR $0.66, P=0.047$ ) were lower in the patients who received bivalirudin alone, compared with their counterparts treated with heparin plus GPI.

The safety and efficacy of bivalirudin monotherapy compared with heparin plus GPI were sustained at threeyear follow-up. Three-year rates of all-cause mortality (5.9\% versus $7.7 \%, P=0.03)$, cardiac mortality $(2.9 \%$ versus $5.1 \%, P=0.001)$, reinfarction $(6.2 \%$ versus $8.2 \%$, $P=0.0001)$, and major bleeding not related to $\mathrm{CABG}$ $(6.9 \%$ versus $10.5 \%, P=0.0001)$ were lower in the bivalirudin group compared with the heparin plus GPI-treated groups. ${ }^{19,20}$

Clinical trial acronyms and their full names are provided in Table 3. Studies demonstrating the adverse outcomes associated with bleeding are summarized in Table 4.

Table 3 Study acronyms and their respective clinical trial full names (in alphabetical order)

\begin{tabular}{|c|c|}
\hline Acronyms & Clinical trial full names \\
\hline ACUITY & Acute Catheterization and Urgent Intervention Triage Strategy \\
\hline CRUSADE & $\begin{array}{l}\text { Can Rapid risk stratification of Unstable angina patients Suppress Adverse outcomes with Early implementation } \\
\text { of the ACC/AHA guidelines }\end{array}$ \\
\hline GRACE & Global Registry of Acute Coronary Events \\
\hline GUSTO & Global Utilization of Streptokinase and Tissue Plasminogen Activator for Occluded Coronary Arteries \\
\hline HORIZONS-AMI & Harmonizing Outcomes with Revascularization and Stents in Acute Myocardial Infarction \\
\hline HORIZONS-SWITCH & Effect of Switching Antithrombin Agents for Primary Angioplasty in Acute Myocardial Infarction \\
\hline OASIS-5 & The Fifth Organization to Assess Strategies in Acute Ischemic Syndrome \\
\hline OASIS-6 & The Sixth Organization to Assess Strategies in Acute Ischemic Syndrome \\
\hline REPLACE - I & Randomized Evaluation in Percutaneous Coronary Intervention Linking Angiomax to Reduced Clinical Events-I \\
\hline REPLACE-2 & Randomized Evaluation in Percutaneous Coronary Intervention Linking Angiomax to Reduced Clinical Events-2 \\
\hline TIMI & Thrombolysis in Myocardial Infarction \\
\hline \multirow[t]{2}{*}{ TRITON-TIMI 38} & Trial to Assess Improvement in Therapeutic Outcomes by Optimizing Platelet Inhibition with \\
\hline & Prasugrel-Thrombolysis in Myocardial Infarction \\
\hline RIVAL & $\begin{array}{l}\text { Radial versus femoral access for coronary angiography and intervention in patients with acute coronary } \\
\text { syndromes }\end{array}$ \\
\hline
\end{tabular}



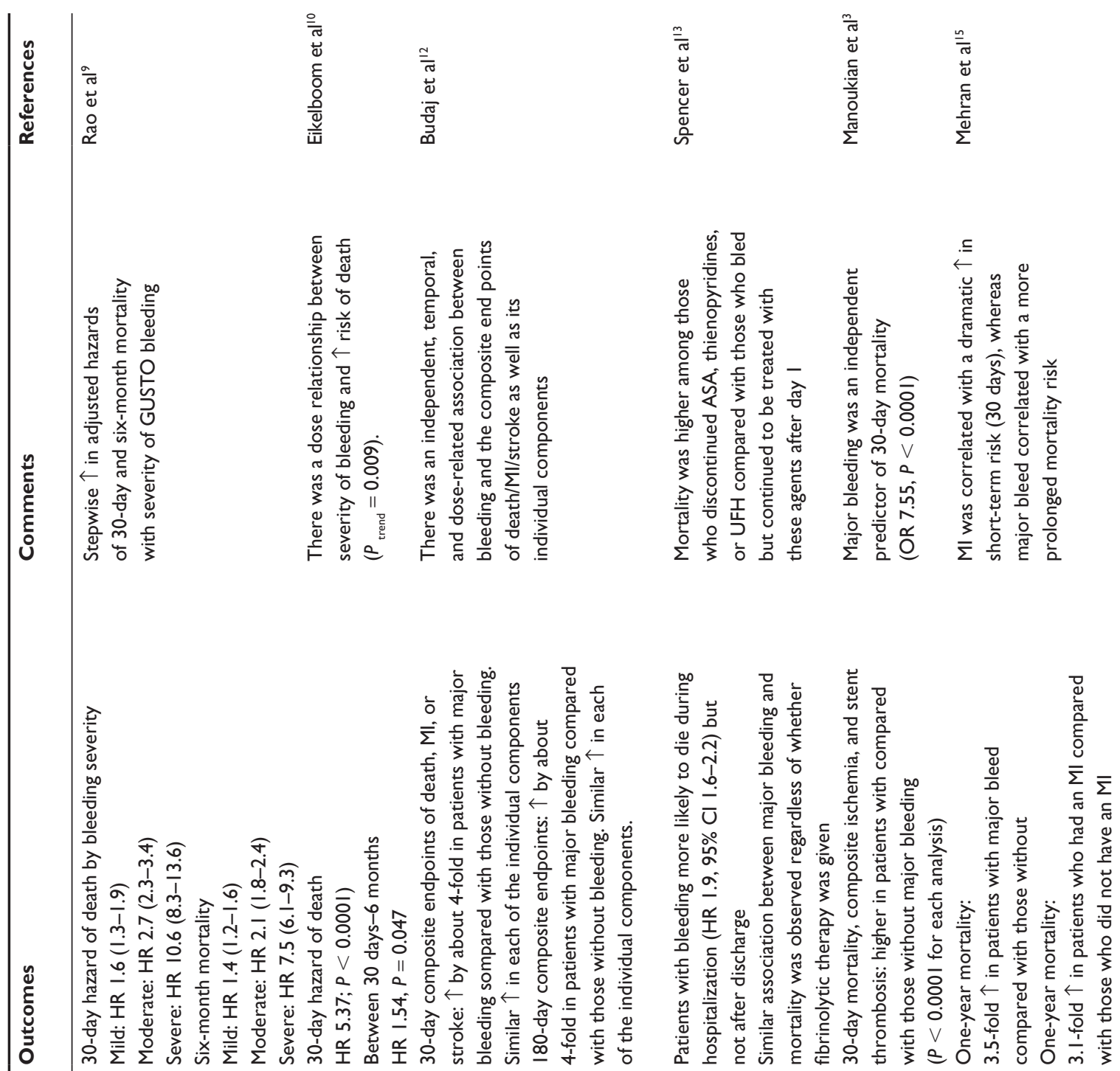

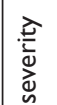

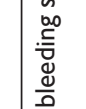

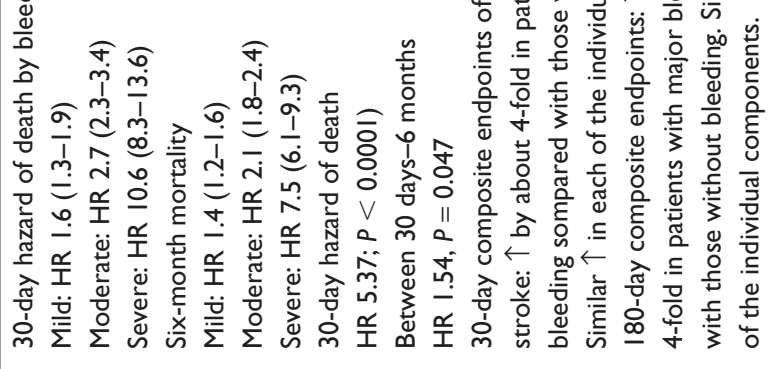
(1)
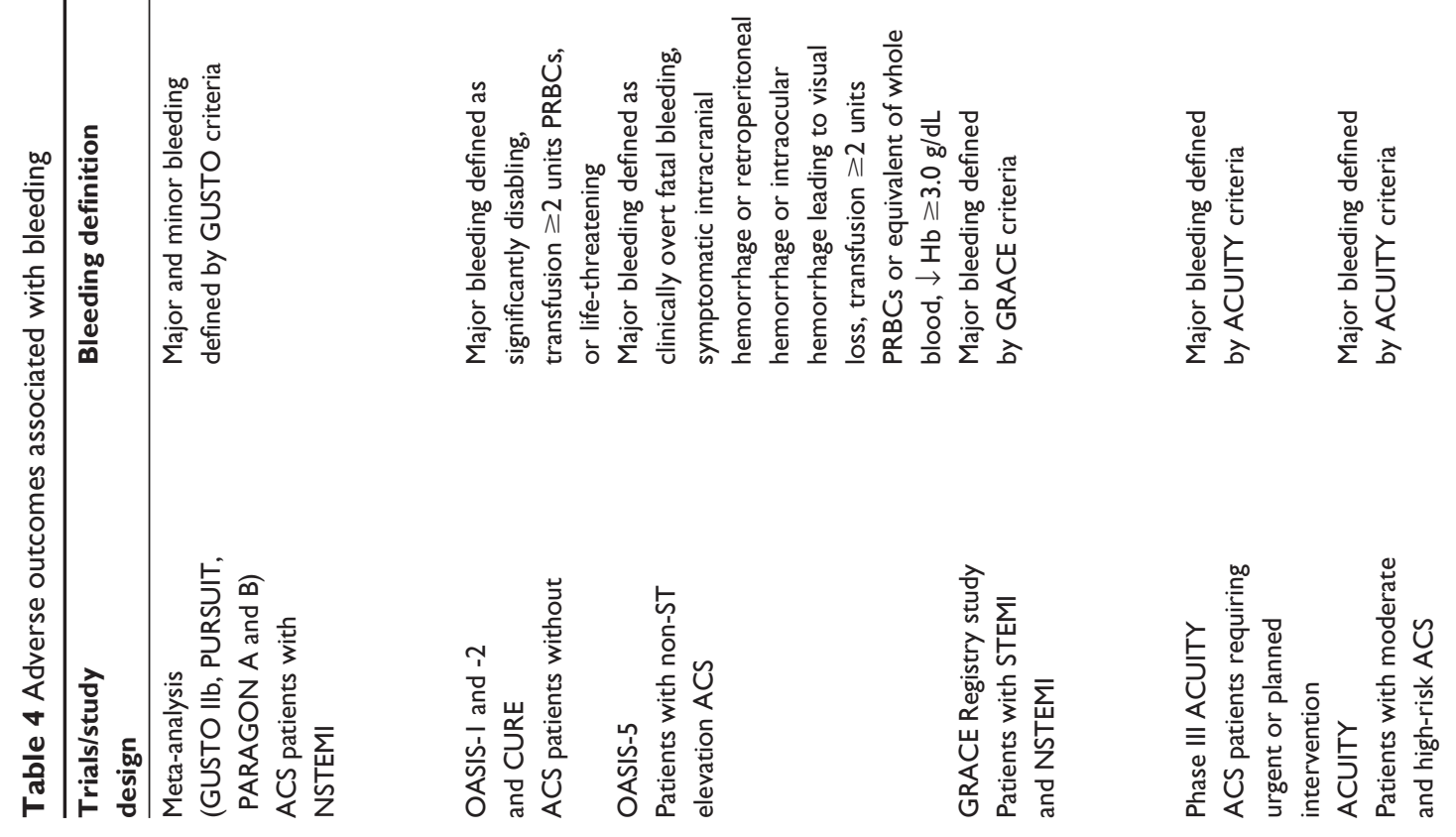

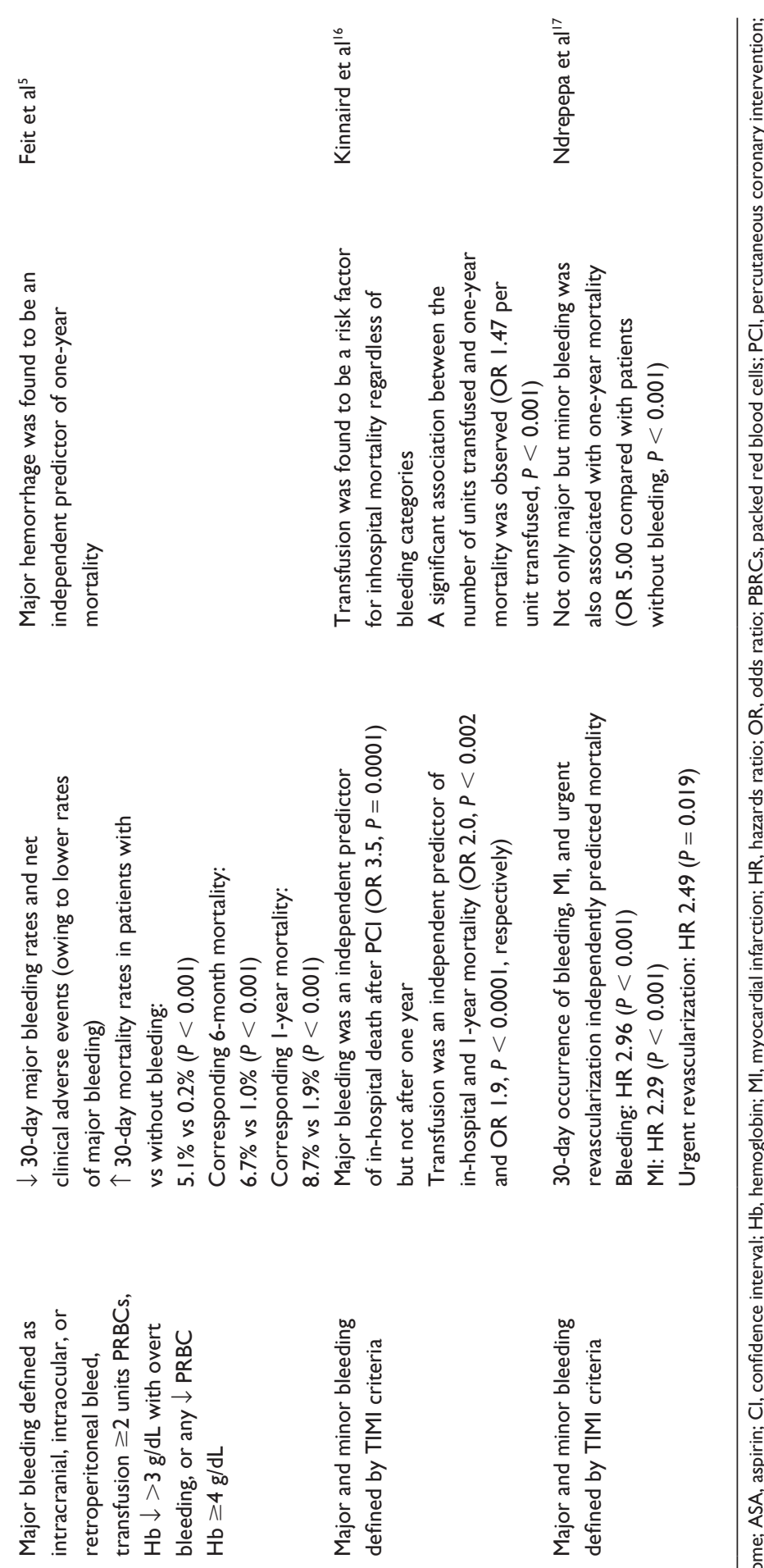

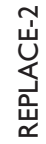
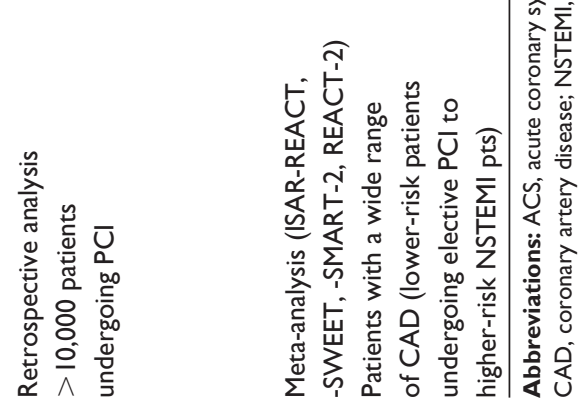
The association between bleeding and adverse outcomes, particularly mortality in patients with ACS and those undergoing PCI, has increasingly been recognized. The difference in the incidence of major bleeding and its associated mortality rates is likely due in part to the lack of a standard definition of bleeding, differences in study design and demographic characteristics, and the potential interplay among various risk factors. Furthermore, patients with bleeding complications commonly have significant comorbidities, which may independently correlate with the risk of nonfatal and fatal adverse outcomes. Despite adjustment for comorbidities, statistical modeling may not capture all baseline differences in demographic characteristics between patients with and those without bleeding. The contributory role of cessation of antiplatelet and antithrombotic therapies to the observed mortality rates in patients with bleeding complications should not be overlooked, and warrants further studies.

\section{Predictive risk factors for bleeding in ACS and PCI}

Bleeding is the most common noncardiac complication of antithrombotic therapy in ACS and PCI. The following section discusses various risk stratification models that have been developed to predict major bleeding in patients with ACS and those undergoing PCI. Study protocol-defined major bleeding is summarized in Table 2 .

Moscucci et al were among the first to devise a prediction rule for identifying patients at higher risk of major bleeding. ${ }^{21}$ In an analysis of the GRACE study, consisting of more than 24,000 patients with ACS, the investigators demonstrated that advanced age, female gender, history of bleeding, and renal insufficiency were independently associated with a higher risk of bleeding $(P<0.01)$. Pharmacological interventions including diuretics, inotropic agents, thrombolytic agents, GPI receptor blockers, and vasodilators were found to be independently associated with an increased risk of bleeding. Similarly, the use of right heart catheterization and PCI were independently associated with an increased risk of bleeding. The predictive factors for major bleeding were similar among the subgroups of patients who presented with a STEMI (4.8\%), NSTEMI (4.7\%), or an unstable angina (2.3\%) event. Further analysis demonstrated that patients with major bleeding had significantly higher inhospital mortality than those without major bleeding (18.6\% versus 5.1\%, $P<0.001)$. After adjustment for comorbidities, clinical presentation and hospital therapies, major bleeding remained independently associated with increased risk of inhospital mortality (OR $1.64,95 \%$ CI 1.18-2.28).
In an analysis of the CRUSADE Quality Improvement Initiative database, Subherwal et al identified eight independent baseline predictors of major inhospital bleeding among community-treated NSTEMI patients. ${ }^{22}$ Of the 89,134 high-risk patients with NSTEMI enrolled in the study, 71,277 patients $(80 \%)$ were randomly assigned to the derivation cohort, and $17,857(20 \%)$ to the validation cohort. Major bleeding occurred in $9.4 \%$ in the derivation cohort and $9.6 \%$ in the validation cohort (not statistically significant for cross-cohort comparisons). Factors independently associated with major bleeding on multivariate analysis included female gender, history of diabetes, prior vascular disease (peripheral vascular disease or stroke), heart rate (higher risk per 10 beats per minute increase), systolic blood pressure (higher risk with pressures above or below 110-180 $\mathrm{mmHg}$ range), signs of congestive heart failure, baseline hematocrit $<36 \%$ (versus $\geq 36 \%$ ), and creatinine clearance (higher risk per $10 \mathrm{~mL} / \mathrm{minute}$ decrease). The CRUSADE major bleeding model derived from the final regression model discriminated patients who did and did not have a major bleeding event in both the derivation $(\mathrm{c}$ statistic $=0.72)$ and validation $(\mathrm{c}$ statistic $=0.71)$ cohorts. Further analysis demonstrated that the CRUSADE bleeding model was similarly able to predict rates of moderate to severe bleeding according to the GUSTO definition (c statistic $=0.71$ ).

To quantify the risk for inhospital major bleeding, a CRUSADE bleeding score was derived by assigning weighted integers to each independent predictor on the basis of its coefficient in the regression model. The bleeding risk was estimated by the sum of the weighted integers (range 1-100 points). Similar to the multivariate model, the CRUSADE bleeding score had a good ability to discriminate between patients who did and did not have a major bleeding event in the derivation (c statistic $=0.71$ ) and validation (c statistic $=0.70$ ) cohorts. In the derivation cohort, the rate of bleeding increased 10 -fold $(<3 \%$ to $>30 \%)$ between patients in the lowest to patients in the highest bleeding scores. A similarly increased trend of major bleeding rates were seen across the quintile risk groups (3.1\% for patients at very low risk [score $\leq 20$ ]; $5.5 \%$ for patients at low risk [score $21-30$ ]; $8.6 \%$ for patients at moderate risk [score 31-40]; $11.9 \%$ for patients at high risk [score 41-50]; and 19.5\% for patients at very high risk [score $>50$ ]; $P_{\text {trend }}<0.001$ ). Similar results were seen across quintiles of risk groups in the validation cohort $\left(P_{\text {trend }}<0.001\right)$. Similar to the CRUSADE major bleeding model, the CRUSADE bleeding score had good ability to discriminate between patients who did and 
did not have a major bleeding event in the derivation and validation cohorts.

Although the CRUSADE bleeding score model did not include postadmission treatments, it demonstrated preserved discrimination across treatment subgroups in the derivation cohort. The incidence of major bleeding was higher among patients who received at least two antithrombotic medications $(n=50,969)$ versus patients who received less than two antithrombotic medications $(n=5931 ; 8.2 \%$ versus $6.9 \%)$. The increased bleeding risk persisted in the analysis within each bleeding risk quintile $\left(P_{\text {trend }}<0.001\right)$. Furthermore, among patients who received at least two antithrombotic medications and underwent invasive procedure (catheterization performed, $n=43,492$ ) versus patients who did not undergo an invasive procedure, the rate of major inhospital bleeding remained higher within each bleeding risk quintile $\left(P_{\text {trend }}<0.001\right)$.

Consistent with the findings of Moscucciet et al, Subherwal et al demonstrated an association between bleeding and inhospital mortality. Patients who experienced a major bleed versus those who did not had a higher mortality rate within each bleeding risk quintile $(2.2 \%$ versus $0.2 \%$ for those at very low risk; $4.4 \%$ versus $0.8 \%$ for those at low risk; $6.9 \%$ versus $1.6 \%$ for those at moderate risk; $9.2 \%$ versus $3.2 \%$ for those at high risk; $11.0 \%$ versus $6.0 \%$ for those at very high risk, $\left.P_{\text {trend }}<0.0001\right)$. A calculation of the CRUSADE bleeding score and its associated risk of inhospital major bleeding is available at www.crusadebleedingscore.org.

Nikolsky et al used the database from two large randomized PCI trials to develop (the REPLACE-2 trial, $n=6002)^{4}$ and validate (the REPLACE- 1 trial, $\mathrm{n}=1056$ ) $^{23}$ a prognostic risk score for major bleeding in patients undergoing elective or urgent PCI via the femoral approach. Similar to the CRUSADE bleeding score, Nikolsky et al found that female gender, baseline anemia, and lower creatinine clearance (estimated glomerular filtration rate $<60 \mathrm{~mL} /$ minute $/ 1.73 \mathrm{~m}^{2}$ ) were independent predictors of bleeding. ${ }^{24}$ Other clinical and procedural variables that were associated with increased risk of bleeding in their bleeding risk model included age $>55$ years, administration of low molecular weight heparin within 48 hours pre-PCI, use of GPI, and intra-aortic balloon pump use. Based on the seven variables identified, a risk score was derived by assigning weighted integers to each independent predictor, the sum of which comprised a total risk score (range $0.9 \%-22.2 \%$ ). Stratification of the risk score into four groups revealed a stepwise increased risk of bleeding with increasing score, with the risk of major bleeding varying from $1.0 \%$ in patients without risk factors to $5.4 \%$ in the high-risk patients (risk score $\geq 10, n=1563$ ). The discriminatory power of the risk model was confirmed in the validation data set (receiving operating curve $=0.62$ ). However, it should be noted that the REPLACE-2 trial enrolled a highly selective population undergoing PCI using the femoral approach, and included treatment variables (ie, invasive procedures and antithrombotics), which limits its utility for assessment of baseline bleeding risk in a community population.

Studies evaluating predictive risk factors for bleeding in ACS and PCI have yielded variable results. Nonetheless, risk factors can be categorized into nonmodifiable and modifiable risks, ie, the former category to identify high-risk individuals, and the latter to optimize treatment variables that are associated with bleeding risks. Commonly reported nonmodifiable risk factors include advanced age, female gender, chronic kidney disease (renal insufficiency), baseline anemia, prior stroke, smaller body weight, history of hypertension or diabetes, and baseline ST segment deviation. Commonly reported modifiable (or potentially modifiable) risk factors include combination therapy with antithrombotic and antiplatelet agents, particularly GPI, large sheath size, invasive procedures (PCI, intra-aortic balloon pump), procedural duration, and femoral versus radial access. The CRUSADE bleeding score permits assessment of baseline bleeding risk, whereas the REPLACE bleeding score permits both baseline and treatment variable risk stratification. Although the CRUSADE bleeding score model did not include postadmission treatments, it is conceivable that bleeding risk increases with increasing number of risk factors.

Suggested predictive risk factors for bleeding in patients with ACS and those undergoing PCI are summarized in Figure 1.

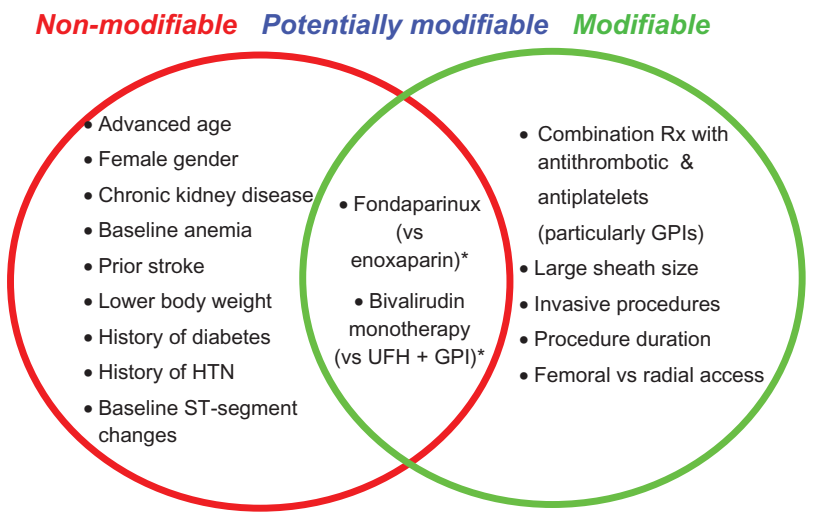

Figure I Risk factors for bleeding

Note: *Discussed under strategies to reduce bleeding.

Abbreviations: HTN, hypertension; UFH, unfractionated heparin; GPIs, glycoprotein inhibitors. 


\section{Mechanisms behind bleeding and mortality}

There has been ample literature suggesting an association between bleeding and mortality in ACS and PCI. Nonetheless, because mortality and bleeding have been demonstrated to have overlapping risk factors, including age, anemia, history of hypertension, renal insufficiency, prior stroke, and baseline ST segment changes, the contributory role of bleeding per se and mortality remains to be defined. The following section discusses the potential mechanisms of the association between bleeding and mortality.

The direct hemodynamic effect of massive hemorrhage on mortality risk and the causal relationship between intracranial bleed with mass effect and mortality appear intuitive. However, the causative role of less severe bleeding on mortality remains speculative. Nonetheless, in acute bleeding, while increased in neurohormonal levels such as norepinephrine, angiotensin, endothelin-1, and vasopressin act to maintain blood pressure and regional blood flow, they have also been demonstrated to be associated with adverse cardiac events. ${ }^{9}$ In hemorrhagic shock, upregulation of cellular adhesion molecules, such as P-selectin and vascular cell adhesion molecule-1, has been suggested to promote eccentric left ventricular remodeling, increase diastolic wall stress, increase oxygen consumption, and thus exacerbates myocardial ischemia and increases mortality. ${ }^{9}$ Furthermore, bleeding can result in reduced oxygen supply and increased oxygen demand, the former attributable to anemia and reduced tissue perfusion and the latter as a result of stress and tachycardia. It is conceivable that the disparity in oxygen supply and demand can lead to coronary ischemia and increased mortality. ${ }^{25}$

In addition to the seemingly direct effect of bleeding on mortality, measures to ameliorate bleeding, such as discontinuation of antithrombotic therapy, paradoxically increases mortality risk. In the GRACE registry study, patients with acute myocardial infarction who experienced bleeding had a higher frequency of discontinuation of aspirin, thienopyridines, and heparin compared with those who did not bleed. ${ }^{13}$ More importantly, Spencer et al demonstrated higher inhospital mortality rates in patients who discontinued aspirin, thienopyridines, or unfractionated heparin after experiencing major bleeding compared with those who continued their anticoagulation therapy despite their bleeding episodes. ${ }^{13}$ Patients with ACS and those undergoing PCI are in a heightened state of thrombosis and platelet activation. It is conceivable that withholding of antithrombotic therapy can result in activation of the coagulation system and platelets, which compromises coronary blood flow leading to myocardial damage, reduced left ventricular function, and fatal cardiac arrhythmias. Furthermore, activation of the coagulation cascade can lead to stent thrombosis and nonfatal and fatal cardiac events. Retrospective analysis of the Phase III ACUITY trial demonstrated a greater than five-fold increase in stent thrombosis among patients who experienced major bleeding. ${ }^{3}$

Acute bleeding may require blood transfusions to replenish plasma volume rapidly and to increase oxygen-carrying capacity for tissue oxygenation. However, there has been accumulating literature demonstrating an association between blood transfusions and increased mortality risk. Results from the GUSTO IIb, PURSUIT, and PARAGON B trials demonstrated that blood transfusions in the setting of ACS were associated with a nearly four-fold increase in the risk of death at 30 days. ${ }^{26,27}$ Transfusion of stored packed red blood cells can result in depletion of 2,3-diphosphoglycerate and nitric oxide from red blood cells resulting in increased affinity of hemoglobin for oxygen and impaired release of oxygen at the capillary level, ultimately leading to reduced tissue oxygen delivery. ${ }^{28,29}$ Hence, a transfusion-associated increase in hemoglobin concentration is not proportionally associated with an increase in tissue oxygenation. ${ }^{30}$ Blood transfusions have also been shown to be associated with an increase in the production of proinflammatory cytokines and other bioreactive substances that may promote coronary ischemia and thrombosis. ${ }^{29,31}$ Other suggested adverse consequences from blood transfusions include exposure to viral pathogens, immune suppression, fluid overload, electrolyte imbalance, and increased blood viscosity. ${ }^{32}$ Lastly, acute bleeding may necessitate invasive interventions and anesthesia which may independently contribute to the increased incidence of mortality observed in these patients. Suggested mechanisms of the association between bleeding and mortality are summarized in Figure 2.

Bleeding can be classified into two arbitrarily defined states, ie, acute, occurring in patients with ACS and those undergoing PCI, and chronic, occurring during maintenance therapy with anticoagulation. The following section discusses suggested strategies to reduce bleeding complications in the acute settings. Discussion of strategies to reduce bleeding complications in the chronic setting is beyond the scope of this article.

\section{Risk stratification}

While no single protocol with maximum treatment efficacy and minimum bleeding risk exists, a preprocedural 


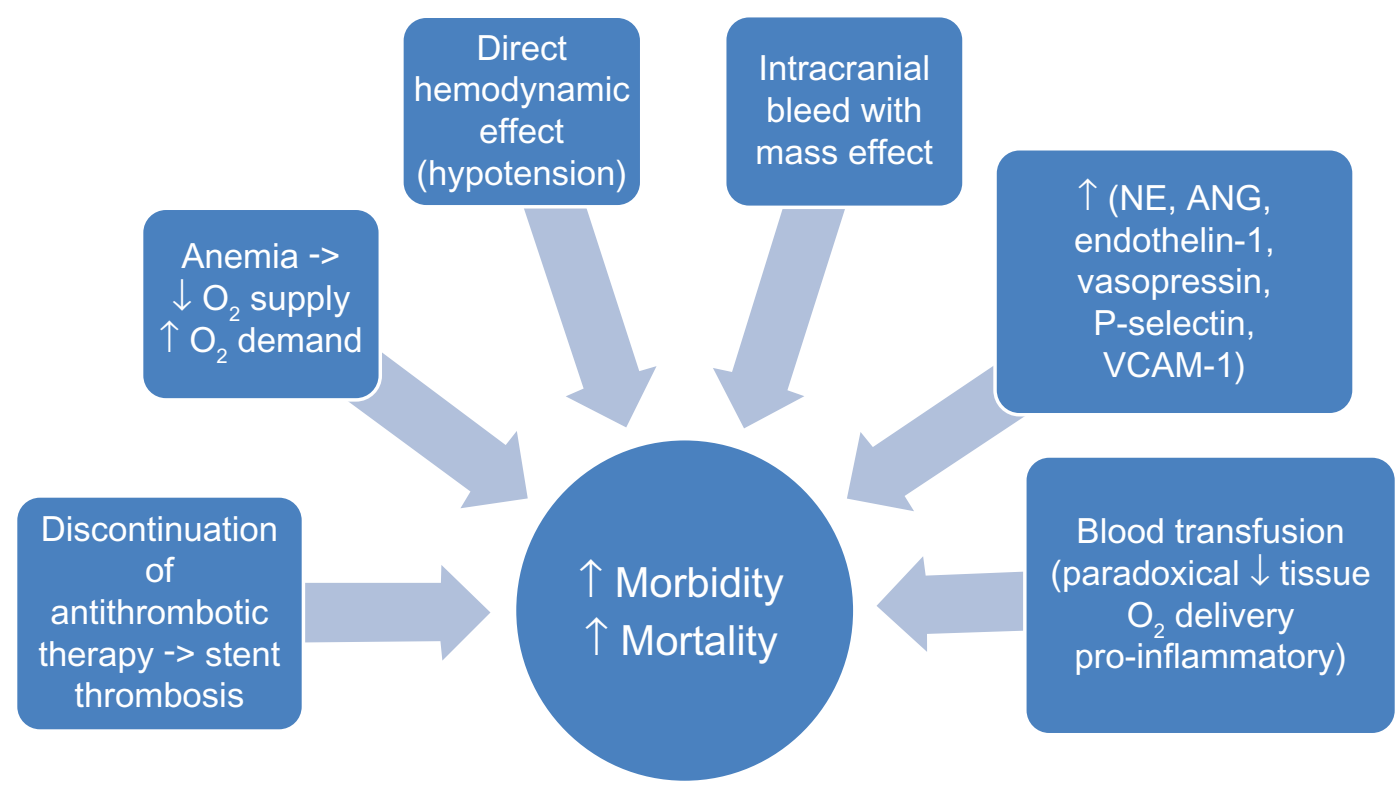

Figure 2 Suggested mechanisms of the association between bleeding and mortality (see text for details). Abbreviations: NE, norepinephrine; ANG, angiotensin, VCAM-I, vascular cell adhesion molecule-I.

risk assessment assists clinicians to tailor procedures and pharmacotherapy to each individual patient. A risk score derived from the REPLACE study demonstrated that the risk of protocol-defined major bleeding varies from $1.0 \%$ in patients without risk factors to greater than $5 \%$ in those with a risk score $>10$, whereas the CRUSADE bleeding score offers baseline risk stratification. $22,23,33$

\section{Choice of antiplatelet and antithrombotic therapy}

Efforts to improve clinical outcomes in patients with ACS and those undergoing PCI entail a judicious balance between antithrombotic effect and risk of bleeding. Hence, unless contraindicated, administration of medications that have been shown to ameliorate bleeding risk should be considered.

The OASIS-5 trial demonstrated that fondaparinux was as effective as enoxaparin in reducing the risk of ischemic events, but with a nearly $50 \%$ reduction in the incidence of major bleeding and a $>60 \%$ reduction in minor bleeding compared with enoxaparin. ${ }^{34}$ More importantly, a reduction in the incidence of bleeding has been suggested to account for the long-term reduction of death in patients treated with fondaparinux compared with their enoxaparin-treated counterparts. Results of both OASIS-5 and OASIS-6 (comparing unfractionated heparin for 4-48 hours versus fondaparinux $2.5 \mathrm{mg}$ subcutaneously daily for up to eight days in a placebo-controlled doubleblind trial in patients with STEMI undergoing PCI) suggest that using unfractionated heparin with fondaparinux during PCI substantially reduces the risk of catheter thrombosis and related complications, without an increase in clinical complications or major bleed. ${ }^{34,35}$ Nonetheless, it should be noted that currently available data do not support a role for fondaparinux during PCI for stable or unstable coronary artery disease.

Although administration of unfractionated heparin is still regarded as the gold standard antithrombotic therapy in the early periprocedural period, bivalirudin overcomes several limitations of heparin and may be considered as an alternative strategy to unfractionated heparin plus GPI, particularly in patients at risk for bleeding complications. Results of the ACUITY and REPLACE-2 trials demonstrated that bivalirudin significantly reduced the risk of bleeding while maintaining efficacy in reducing ischemic events in comparison with heparin plus GPI.,4 Similarly, the HORIZONS-AMI trial demonstrated reduced rates of net adverse clinical events and major bleeding with the use of bivalirudin monotherapy compared with heparin plus GPI in patients with STEMI undergoing PCI. ${ }^{19,36}$ However, the use of bivalirudin is relatively contraindicated in patients with chronic total occlusion because there is currently no agent to reverse the antithrombotic effect of bivalirudin. Bivalirudin should be used with caution in patients without unfractionated heparin or clopidogrel pretreatment due to increased risk of acute stent thrombosis.

In a recent analysis of the HORIZONS-SWITCH study, Dangas et al demonstrated that in patients with STEMI 
who received early treatment with unfractionated heparin, switching to bivalirudin before primary PCI resulted in reduced rates of major bleeding and improved early and late cardiac survival. ${ }^{37}$ Study subjects consisted of 2357 patients in the HORIZONS-AMI trial who received unfractionated heparin before study enrollment and were subsequently randomly assigned to bivalirudin (switch group, $\mathrm{n}=1178$ ) or unfractionated heparin plus GPI (control group, $\mathrm{n}=1179$ ). At 30 days, major bleeding occurred in $7.6 \%$ and $12.3 \%$ in the switch and control groups, respectively, $P=0.0001$. Furthermore, 30-day rates of cardiac mortality was lower in the switch compared with the control groups (1.6\% versus $2.9 \%$, respectively, $P=0.04$ ). Similarly, at two-year follow-up, switch patients experienced lower rates of major bleeding $(P=0.0003)$, cardiac mortality $(P=0.04)$, and reinfarction $(P=0.0002)$ while two-year rates of definite or probable stent thrombosis were similar between the two treatment groups (switch versus control, 3.1\% versus $4.3 \%$, respectively, $P=0.17$ ). The results of the study suggested that a strategy of switching STEMI patients who received early treatment with unfractionated heparin to bivalirudin may be safe and effective, resulting in reduced hemorrhagic complications and cardiac mortality and enhanced event-free survival compared with unfractionated heparin continuation and initiation of GPI. Nonetheless, the investigators acknowledged that the results of the HORIZON-SWITCH study should be considered exploratory and hypothesisgenerating due to limited statistical power. Further studies are needed.

Results of the TRITON-TIMI 38 trial demonstrated that patients with ACS and known coronary anatomy who received aspirin and prasugrel had a lower risk of ischemic event but a greater risk of TIMI major and life-threatening bleeding (particularly in those undergoing CABG or those with a history of cerebrovascular disease) compared with those receiving aspirin and clopidogrel. ${ }^{38,39}$ Further analysis

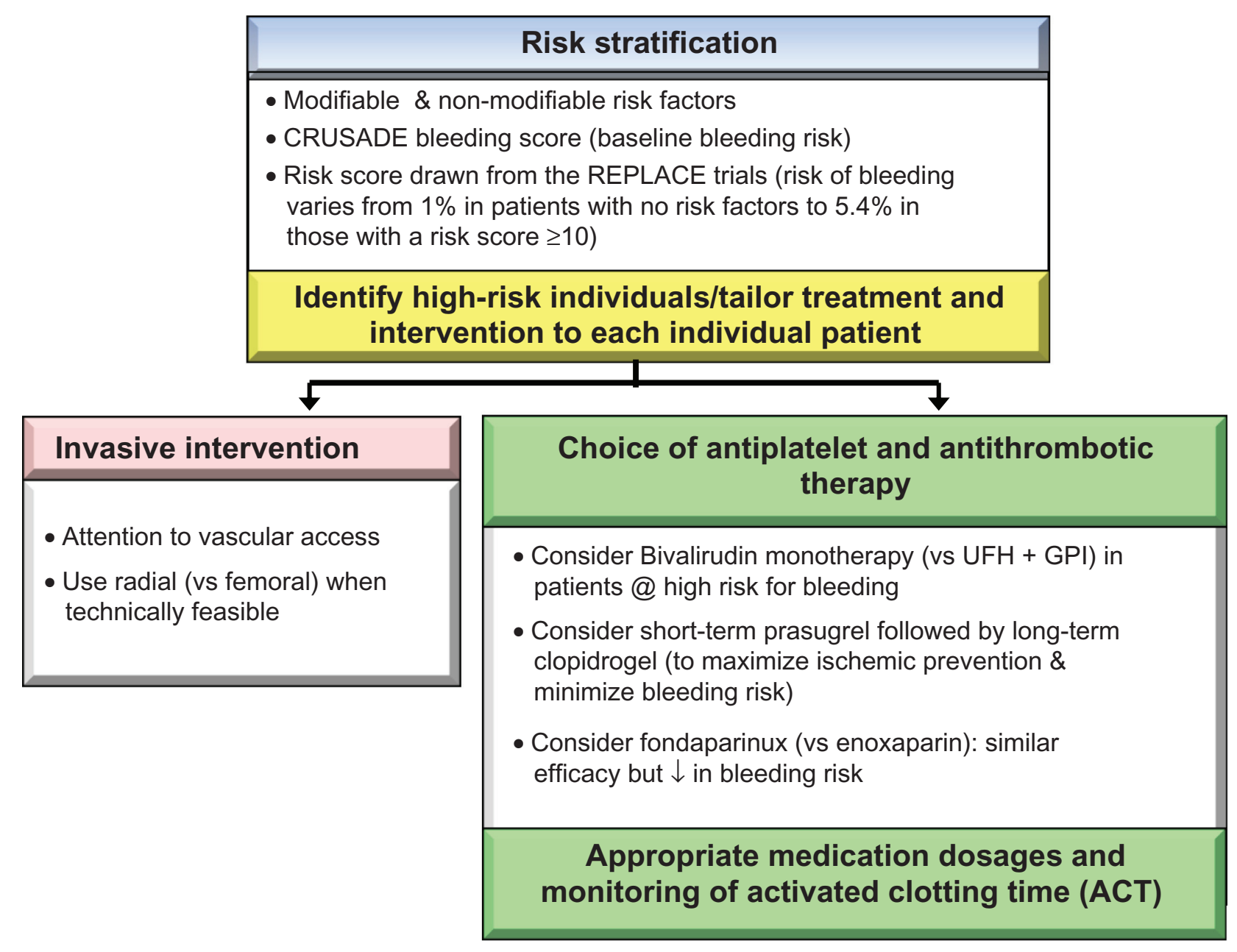

Figure 3 Suggested strategies to reduce bleeding complications (see text for details). 
demonstrated that the majority of ischemic event reduction associated with prasugrel occurs during the first 30 days of treatment while bleeding events continue to accrue during long-term maintenance therapy. Hence, it has been suggested that one strategy to minimize bleeding while maximizing ischemic protection might involve the early use of prasugrel in patients with ACS undergoing PCI, followed by long-term therapy with clopidogrel. Such strategy might also reduce the rates of bleeding-related discontinuation of therapy, which was significantly greater among prasugrel versus clopidogrel-treated patients. The routine recommendation of short-term prasugrel followed by long-term clopidogrel to maximize treatment efficacy and minimize bleeding risk awaits further studies. Nonetheless, it should be noted that prasugrel is contraindicated in patients with active pathological bleeding, history of stroke or transient ischemic attack, and those who will likely require coronary artery bypass grafting.

\section{Medication dosages and laboratory monitoring}

The CRUSADE registry database revealed that over $42 \%$ of patients with NSTEMI received one or more antithrombotic agents above the recommended dosage range. ${ }^{40}$ Excessive dosing and the number of agents administered in excess were found to be directly related to the risks of bleeding. It has been suggested that proper dosing requires adjustment based on body weight and renal function. Meticulous attention to appropriate dosing of antithrombotic agents, particularly GPI and unfractionated heparin, and close monitoring of activated clotting time may reduce bleeding risks. Suggested activated clotting time for patients undergoing PCI with and without GPI are 200-250 seconds and 300-350 seconds, respectively.

\section{Invasive intervention}

In addition to manipulating antithrombotic strategies, reduction of bleeding risk can also be achieved with careful attention to vascular access, using the radial artery approach (versus femoral) during cardiac catheterization and PCI procedures, and using vascular closure devices post cardiac catheterization and post-PCI. However, the use of vascular closure devices to reduce vascular bleeding remains controversial, except for the benefit of decreasing time to ambulation and length of hospitalization. Currently, the American Heart Association issues a Class III recommendation of use of vascular closure devices for the purpose of reducing vascular complications. Nonetheless, the rates of bleeding complications or procedural failures or both may be operatordependent and the choice of the procedure should be at the discretion of the interventional cardiologists. ${ }^{39,41-44}$ Suggested strategies to reduce bleeding complications are summarized in Figure 3.

\section{Conclusion}

The advent of potent antiplatelet and antithrombin agents has resulted in significant improvement in cardiovascular outcomes in patients with ACS and those undergoing PCI. However, these treatment strategies have led to an increase in the risk of bleeding. Such bleeding complications have been shown to be associated with myocardial infarction, stroke risk, and more importantly mortality. Hence, assessment of bleeding risks and implementing pharmacological and nonpharmacological measures to reduce bleeding complications should be an integral part of the management of patients with ACS and those undergoing PCI. Appropriate dosing of medications should not be overlooked. The judicious balance between antithrombotic effect and bleeding risk and the use of the radial approach when technically feasible may improve clinical outcomes. Intervention aimed at halting the bleeding may paradoxically have a negative impact on outcomes, hence management of periprocedural bleeding should be individualized. While the association between blood transfusions and mortality has not been fully elucidated, blood transfusion should only be given after carefully weighing the risks and benefits. Lastly, future clinical trials should include bleeding as a quadruple primary endpoint, combining death, myocardial infarction, urgent repeat revascularization, and bleeding to better assess patients' cardiovascular outcomes.

\section{Disclosure}

The authors report no conflicts of interest in this work.

\section{References}

1. Chesebro JH, Knatterud G, Poberts R, et al. Thrombolysis in Myocardial Infarction (TIMI) trial, Phase I: A comparison between intravenous tissue plasminogen activator and intravenous streptokinase. Clinical findings through hospital discharge. Circulation. 1987;76:142-154.

2. The GUSTO Investigators. An international randomized trial comparing four thrombolytic strategies for acute myocardial infarction. $N$ Engl J Med. 1993;329:673-682.

3. Manoukian SV, Feit F, Mehran R, et al. Impact of major bleeding on 30-day mortality and clinical outcomes in patients with acute coronary syndromes. An analysis from the ACUITY trial. J Am Coll Cardiol. 2007;49:1362-1368. 
4. Lincoff AM, Bittl JA, Harrington RA, et al. Bivalirudin and provisional glycoprotein IIb/IIIa blockade compared with heparin and planned glycoprotein IIb/IIIa blockade during percutaneous coronary intervention. REPLACE-2 randomized trial. JAMA. 2003;289: 853-863.

5. Feit F, Voeltz MD, Attubato MJ, et al. Predictors and impact of major hemorrhage on mortality following percutaneous coronary intervention from the REPLACE-2 trial. Am J Cardiol. 2007;100:1364-1369.

6. Steg PG, Huber K, Andreotti F, et al. Bleeding in acute coronary syndromes and percutaneous coronary interventions: Position paper by the Working Group on Thrombosis of the European Society of Cardiology. Eur Heart J. 2011;32:1854-1864.

7. Mehran R, Rao S, Bhatt DL, et al. Standardized bleeding definitions for cardiovascular clinical trials. A consensus report from the Bleeding Academic Research Consortium. Circulation. 2011;123: 2736-2747.

8. Rao SV, O'Grady K, Pieper KS, et al. A comparison of the clinical impact of bleeding measured by two different classifications among patients with acute coronary syndromes. $\mathrm{J} \mathrm{Am} \mathrm{Coll} \mathrm{Cardiol.}$ 2006;47:809-816.

9. Rao SV, O'Grady K, Pieper KS, et al. Impact of bleeding severity on clinical outcomes among patient with acute coronary syndromes. Am J Cardiol. 2005;96:1200-1206.

10. Eikelboom JW, Mehta SR, Anand SS, et al. Adverse impact of bleeding on prognosis in patients with acute coronary syndromes. Circulation. 2006;114:774-782.

11. Yusuf S, Phil D, Mehta SR, et al. Comparison of fondaparinux and enoxaparin in acute coronary syndromes. The Fifth Organization to Assess Strategies in Acute Ischemic Syndromes (OASIS-5). $N$ Engl $J$ Med. 2006;354:1464-1476.

12. Budaj A, Eikelboom JW, Mehta SR, et al. Improving clinical outcomes by reducing bleeding in patients with non-ST-elevation acute coronary syndromes. Eur Heart J. 2009;30:655-661.

13. Spencer FA, Moscucci M, Granger CB, et al. Does comorbidity account for the excess mortality in patients with major bleeding in acute myocardial infarction? Circulation. 2007;116:2793-2801.

14. Stone GW, McLaurin BT, Cox DA, et al. Bivalirudin for patients with acute coronary syndromes. $N$ Engl J Med. 2006;355:2203-2216.

15. Mehran R, Pocock SJ, Stone GW, et al. Associations of major bleeding and myocardial infarction with the incidence and timing of mortality in patients presenting with non-ST-elevation acute coronary syndromes: A risk model from the ACUITY trial. Eur Heart $J$. 2009;30:1457-1466.

16. Kinnaird TD, Stabile E, Mintz GS, et al. Incidence, predictors, and prognostic implications of bleeding and blood transfusion following percutaneous coronary interventions. Am J Cardiol. 2003;92:930-935.

17. Ndrepepa G, Berger PB, Mehilli J, et al. Periprocedural bleeding and 1-year outcome after percutaneous coronary interventions. J Am Coll Cardiol. 2008;51:690-697.

18. Stone GW, Witzenbichler B, Guagliumi G, et al. Bivalirudin during primary PCI in acute myocardial infarction. $N$ Engl $J$ Med. 2008;358:2218-2230.

19. Mehran R, Lansky AJ, Witzenbichler B, et al. Bivalirudin in patients undergoing primary angioplasty for acute myocardial infarction (HORIZONS-AMI): 1-year results of a randomized controlled trial. Lancet. 2009;374:1149-1159.

20. Stone GW, Witzenbichler B, Guagliumi G, et al. Heparin plus a glycoprotein IIb/IIIa inhibitor versus bivalirudin monotherapy and paclitaxel-eluting stents versus bare-metal stents in acute myocardial infarction (HORIZONS-AMI): Final 3-year results from a multicentre, randomized controlled trial. Lancet. 2011;377: 2193-2204.

21. Moscucci M, Fox KAA, Cannon CP, et al. Predictors of major bleeding in acute coronary syndromes: The Global Registry of Acute Coronary Events (GRACE). Eur Heart J. 2003;24:1815-1823.
22. Subherwal S, Bach RG, Chen AY, et al. Baseline risk of major bleeding in non-ST-segment-elevation myocardial infarction. The CRUSADE (Can Rapid risk stratification of Unstable angina patients Suppress ADverse outcomes with Early implementation of the ACC/AHA guidelines) bleeding score. Circulation. 2009;119:1873-1882.

23. Lincoff AM, Bittl JA, Kleiman NS, et al. Comparison of bivalirudin versus heparin during percutaneous coronary intervention (the Randomized Evaluation of PCI Linking Angiomax to Reduced Clinical Events [REPLACE]-1 Trial). Am J Cardiol. 2004;93:1092-1096.

24. Nikolsky E, Mehran R, Dangas G, et al. Development and validation of a prognostic risk score for major bleeding in patients undergoing percutaneous coronary intervention via the femoral approach. Eur Heart J. 2007;28:1936-1945.

25. Sabatine MS, Morrow DA, Guigliano RP, et al. Association of hemoglobin levels with clinical outcomes in acute coronary syndromes. Circulation. 2005;111:2042-2049.

26. Rao SV, Jollis JG, Harrington RA, et al. Relationship of blood transfusion and clinical outcomes in patients with acute coronary syndromes. JAMA. 2004;292:1555-1562.

27. Guitierrez A, Rao SV. Incidence, outcomes, and management of bleeding in non-ST-elevation acute coronary syndromes. Cleve Clin J Med. 2010;77:369-379

28. Robinson SD, Janssen C, Fretz EB, et al. Red blood cell storage duration and mortality in patients undergoing percutaneous coronary intervention. Am Heart J. 2010;159:876-881.

29. Eikelboom JW, Cook RJ, Liu Y, et al. Duration of red cell storage before transfusion and in-hospital mortality. Am Heart J. 2010;159: 737-743.e1.

30. Rao SV, Califf RM. Is old blood bad blood? Am Heart J. 2010;159: $710-712$.

31. Aronson D, Dann EJ, Bonstein L, et al. Impact of red blood cell transfusion on clinical outcomes in patients with acute myocardial infarction. Am J Cardiol. 2008;102:115-119.

32. Yang X, Alexander KP, Chen AY, et al. The implications of blood transfusions for patients with non-ST-segment elevation acute coronary syndromes. Results from the CRUSADE national quality improvement initiative. J Am Coll Cardiol. 2005;46:1490-1495.

33. Mehran R, Pocock SJ, Nikolsky E, et al. A risk score to predict bleeding in patients with acute coronary syndromes. $J$ Am Coll Cardiol. 2010;55:2556-2566.

34. Mehta SR, Granger CB, Eikilboom JW, et al. Efficacy and safety of fondaparinux versus enoxaparin in patients with acute coronary syndromes undergoing percutaneous coronary intervention. Results from the OASIS-5 trial. J Am Coll Cardiol. 2007;50:1742-1751.

35. Yusuf S, Mehta SR, Chrolavicius S, et al. Effects of fondaparinux on mortality and reinfarction in patients with acute ST-segment elevation myocardial infarction. The OASIS-6 randomized trial. JAMA. 2006;295:1519-1530.

36. Pham SV, Pham PC, Pham PM, et al. Antithrombotic strategies in patients undergoing percutaneous coronary intervention for acute coronary syndrome. Drug Des Devel Ther. 2010;4:203-220.

37. Dangas GD, Mehran R, Nikolsky E, et al. Effect of switching antithrombotic agents for primary angioplasty in acute myocardial infarction. The HORIZONS-SWITCH analysis. $\mathrm{J} \mathrm{Am} \mathrm{Coll} \mathrm{Cardiol.}$ 2011;57:2309-2316.

38. Montalescot G, Wiviott SD, Braunwald E, et al. Prasugrel compared with clopidogrel in patients undergoing percutaneous coronary intervention for ST-elevation myocardial infarction (TRITON-TIMI 38): Doubleblind, randomized controlled trial. Lancet. 2009;373:723-731.

39. Manoukian SV. The relationship between bleeding and adverse outcomes in ACS and PCI: Pharmacological and nonpharmacological modification of risk. J Invasive Cardiol. 2010;22:132-141.

40. Alexander KP, Chen AY, Roe MT, et al. Excess dosing of antiplatelet and antithrombotic agents in the treatment of non-ST-segment elevation acute coronary syndromes. JAMA. 2005;294:3108-3116. 
41. Rao SV. Strategies to reduce bleeding among patients with ischemic heart disease treated with antiplatelet therapies. Am J Cardiol. 2009;104 Suppl:60-3C.

42. Jolly SS, Amlani S, Hamon M, et al. Radial versus femoral access for coronary angiography or intervention and the impact on major bleeding and ischemic events: A systematic review and meta-analysis of randomized trials. Am Heart J. 2009;157:132-140.
43. Jolly SS, Yusuf S, Cairns J, et al. Radial versus femoral access for coronary angiography and intervention in patients with acute coronary syndromes (RIVAL): A randomized, parallel group, multicentre trial. Lancet. 2011;377:1409-1420.

44. Dauerman HL, Rao SV, Resnic FS, et al. Bleeding avoidance strategies. Consensus and controversy. J Am Coll Cardiol. 2011;58:1-10.

\section{Publish your work in this journal}

Vascular Health and Risk Management is an international, peerreviewed journal of therapeutics and risk management, focusing on concise rapid reporting of clinical studies on the processes involved in the maintenance of vascular health; the monitoring, prevention and treatment of vascular disease and its sequelae; and the involvement of metabolic disorders, particularly diabetes. This journal is indexed on PubMed Central and MedLine. The manuscript management system is completely online and includes a very quick and fair peer-review system, which is all easy to use. Visit http://www.dovepress.com/ testimonials.php to read real quotes from published authors.

Submit your manuscript here: http://www.dovepress.com/vascular-health-and-risk-management-journal 